\title{
Existence of blow-up solutions for quasilinear elliptic equation with nonlinear gradient term. ${ }^{1}$
}

\author{
FANG LI \\ Institute of Mathematics, School of \\ Mathematical Sciences, \\ Nanjing Normal University, \\ Jiangsu Nanjing 210023, China. \\ lifang1012160126. com
}

\author{
ZUODONG YANG \\ School of Teacher Education, \\ Nanjing Normal University, \\ Jiangsu Nanjing 210097, China \\ zdyang-jin@263.net.
}

\begin{abstract}
In this paper, we consider the quasilinear elliptic equation in a smooth bounded domain. By using the method of lower and upper solutions, we study the existence, asymptotic behavior near the boundary and uniqueness of the positive blow-up solutions for quasilinear elliptic equation with nonlinear gradient term.
\end{abstract}

\section{RESUMEN}

En este artículo consideramos la ecuación elíptica cuasilineal en un dominio acotado suave. Usando el método de sub y súper soluciones, estudiamos la existencia, comportamiento asintótico cerca de la frontera y la unicidad de soluciones explosivas para ecuaciones elípticas cuasilineales con término del gradiente nolineal.

Keywords and Phrases: Quasilinear elliptic equation; Blow-up solutions; Asymptotic behavior of solutions; Lower and upper solutions.

2010 AMS Mathematics Subject Classification: 35J65, 35J50.

\footnotetext{
${ }^{1}$ Project Supported by the National Natural Science Foundation of China(No.11171092); the Natural Science Foundation of the Jiangsu Higher Education Institutions of China(No.08KJB110005)
} 


\section{Introduction and main results}

We shall establish the results on the existence, asymptotic behavior near the boundary and uniqueness near the boundary for the following quasilinear elliptic equation

$$
\begin{cases}\triangle_{\mathfrak{m}} \mathrm{u}=\mathrm{b}(\mathrm{x}) \mathrm{u}^{\mathrm{p}}\left(1+|\nabla u|^{\mathfrak{q}}\right), & x \in \Omega, \\ \mathrm{u}=\infty, & x \in \partial \Omega,\end{cases}
$$

where $\Omega$ is a $C^{2}$ bounded domain with smooth boundary $\partial \Omega$ in $\mathbf{R}^{\mathrm{N}}, \triangle_{\mathrm{m}} \mathbf{u}:=$ $\operatorname{div}\left(|\nabla u|^{m-2} \nabla u\right), m \geq 2, p, q>0, b(x) \in C^{\mu}(\bar{\Omega})$ for some $0<\mu<1$.

Problems like (1.1) are usually known in the literature as a boundary blow-up problems and its solutions are named "blow-up solutions" or "explosive solutions" or "large solutions" of Eq. (1.1). Precisely, by a solution of (1.1) we mean a solution of (1.1) satisfying $u(x) \rightarrow \infty$ as $d(x, \partial \Omega) \rightarrow 0$.

Semilinear elliptic problems involving a gradient term with boundary blow-up interested many authors. Namely Bandle and Giarrusso[1] developed existence and asymptotic behavior results for large solutions of $\Delta u+|\nabla u(x)|^{a}=g(u)$ in a bounded domain. In the case $g(u)=p(x) u^{\gamma}, a>0$, and $\gamma>\max (1, a)$. Ghergu et al.[2] considered more general equation $\Delta u+q(x)|\nabla u(x)|^{a}=p(x) g(u)$, where $0 \leq a \leq$ 2, $p$ and $q$ are Hölder continuous functions on $(0, \infty)$. More results about some extensions to this problems, we can see in[24]-[25].

Recently, Goncalves et al. [11] showed the existence of nonnegative solutions of the boundary blow-up problem

$$
\begin{cases}\triangle u=\psi(x, u, \nabla u), & x \in \Omega, \\ u=\infty, & x \in \partial \Omega\end{cases}
$$

under the condition

$$
a(x) g(t) \leq \psi(x, t, \xi) \leq h(t)\left(1+\Lambda|\xi|^{2}\right),
$$

where $\Lambda>0$ is a constant, $a, g$ and $h$ are continuous functions, $a(x)>0$ in $\Omega, g$ and $h$ are non-decreasing and satisfying $g(0)=0, g(t)>0$ for $t>0, h(0) \geq 1$, and $\mathrm{g}$ satisfies the so called Keller-Osserman condition, namely

$$
\int_{1}^{\infty} \frac{1}{\sqrt{G(t)}} d t<\infty, \quad G(t)=\int_{0}^{t} g(s) d s .
$$

The study of the following equation:

$$
\left\{\begin{array}{l}
\triangle_{\mathrm{m}} \mathrm{u}=\mathrm{g}(\mathrm{x}) \mathrm{f}(\mathrm{u}), \quad \text { in } \Omega \\
\mathrm{u}(\mathrm{x}) \rightarrow \infty, \quad \text { as } \quad x \rightarrow \partial \Omega
\end{array}\right.
$$


also has been many results, see [3],[4],[15]-[19] and the references therein. Gladiali and Porru [15] studied boundary asymptotic of solutions of this equation under some condition on $f$ and when $g(x) \equiv 1$. Related problems on asymptotic behavior and uniqueness were also studied in [16]. Ahmed Mohammed in [17] established boundary asymptotic estimate for solution of this equation under appropriate conditions on $g$ and the nonlinearity $f$. They still allowed $g$ to be unbounded on $\Omega$ or to vanish on $\partial \Omega$. Diaz and Letelier [18] proved the existence and uniqueness of large solutions to the problem (1.3) both for $f(u)=u^{\gamma}, \gamma>\mathfrak{m}-1$ (super-linear case) and $\partial \Omega$ being of the class $\mathrm{C}^{2}$. Lu, Yang and E.H.Twizell [4] proved the existence of Large solutions to the problem (1.1) both for $f(u)=u^{\gamma}, \gamma>m-1, \Omega=\mathbf{R}^{N}$ or $\Omega$ being a bounded domain (super-linear case) and $\gamma \leq \mathrm{m}-1, \Omega=\mathbf{R}^{\mathrm{N}}$ (sub-linear case) respectively. Z.Yang et.al. [19] also established an explosive sub-supersolution method for the existence of solutions to (1.3).

For the other results of large solutions to quasilinear elliptic problems (1.1) with nonlinear gradient terms, see [5]-[8] and the references therein.

Motivated by the results of the above cited papers, we shall attempt to treat such equation (1.1), the results of the semilinear equations are extended to the quasilinear ones. We can find the related results for $m=2$ in [10].

To study (1.1),we first consider the existence of nonnegative solutions of the generary boundary blow-up problem

$$
\begin{cases}\triangle_{\mathfrak{m}} u=\psi(x, u, \nabla u), & x \in \Omega, \\ u=\infty, & x \in \partial \Omega .\end{cases}
$$

Our main results are summarized in the following and to our best knowledge, they are not covered by any of the ones referred to above.

Theorem 1.1. Let $\psi \in \mathrm{C}^{\mu}\left(\Omega \times \mathbb{R} \times \mathbf{R}^{\mathrm{N}}\right), 0<\mu<1$, and $\overline{\mathrm{u}}, \underline{u} \in \mathrm{W}^{1, \infty}(\Omega)$ be the ordered weak upper and lower solutions of (1.4) and be bounded on any closed subdomain of $\Omega$. Assume that there exist constants $k>m-1, c_{1}>0$, and two functions $h_{1} \in \mathrm{C}^{\mu}(\Omega)$ and $g \in \mathrm{L}_{\mathrm{loc}}^{\infty}([0,+\infty))$ for some $0<\mu<1$, such that

$$
|\psi(x, t, \xi)| \leq h_{1}(x)+g(t)+c_{1}|\xi|^{k-1} \text { a.e. } x \in \Omega, \forall \xi \in \mathbf{R}^{N}, t \in[\underline{u}, \bar{u}] .
$$

Then there is a $C^{1, \beta}(\Omega)$-solution $u$ of (1.4) for some $0<\beta<1$ such that $\underline{u} \leq \boldsymbol{u} \leq \bar{u}$ in $\Omega$.The ordered weak upper and lower solutions will be defined by Definition 2.1 .

Theorem 1.2. Suppose that $m \geq 2, p, q>0$ and $p+q>m-1$. If there exist two constants $\gamma \geq 0$ and $\beta_{1}>0$ such that $\gamma+m-q \geq 0$ and $b(x) \geq \beta_{1} d^{\gamma}(x)$. Then the problem (1.1) has at least one nonnegative $C^{1}$-solution.

Theorem 1.3. Suppose that $b(x)>0$ in $\Omega, m \geq 2, p, q>0$ and $p+q>m-1$. 
If there are two constants $\beta>0$ and $\gamma \geq 0$ satisfying $\gamma+m-q>0$, such that

$$
\lim _{d(x) \rightarrow 0} \frac{b(x)}{d(x)^{\gamma}}=\beta .
$$

Then the problem (1.1) possesses a nonnegative solution and any nonnegative solution $u(x)$ satisfies

$$
\lim _{d(x) \rightarrow 0} \frac{u(x)}{d^{-\alpha}(x)}=\left(\frac{\alpha^{\mathfrak{m}-1-q}(\alpha+1)(m-1)}{\beta}\right)^{\frac{1}{p+q+1-m}},
$$

where $\alpha=\frac{\gamma+m-q}{p+q-m+1}$. Furthermore, if $p \geq m-1$, then the nonnegative solution of (1.1) is unique.

This work is organized as follows: In Section 2, we give a comparison principle and prove Theorem 1.1. In Section 3, we first find out the blow-up rate in the radially symmetric case and then prove Theorems 1.2 and 1.3 .

\section{Proofs of Theorem 1.1}

Firstly, we consider the second order quasilinear operator Q of the form:

$$
\mathrm{Q}(\mathfrak{u}, \varphi)=\int_{\Omega}(\mathrm{A}(\mathrm{x}, \mathfrak{u}, \nabla \mathfrak{u}) \cdot \nabla \varphi-\mathrm{b}(x, \mathfrak{u}, \nabla \mathfrak{u}) \varphi) \mathrm{d} x
$$

where $x=\left(x_{1}, \ldots, x_{n}\right)$ is contained in the domain $\Omega$ of $\mathbf{R}^{\mathrm{N}}$, the functions $A(x, z, p)$ and $b(x, z, p)$ are assumed to be defined for all values of $(x, z, p)$ in the set $\Omega \times \mathbf{R} \times$ $\mathbf{R}^{\mathrm{N}}, \varphi \in \mathrm{C}_{0}^{\infty}(\Omega)$.

From [23], we get the following comparison principle which plays an important role in the proofs of Theorems 1.2 and 1.3.

Lemma 2.1.(Comparison principle) Let $u, v \in C^{1}(\bar{\Omega})$ satisfy $Q u \geq 0$ in $\Omega$, $\mathrm{Q} v \leq 0$ in $\Omega$ and $u \leq v$ on $\partial \Omega$, where the functions $A$, b are continuously differentiable with respect to the $z$, p variables in $\bar{\Omega} \times \mathbf{R} \times \mathbf{R}^{\mathrm{N}}$, the operator $\mathrm{Q}$ is elliptic in $\Omega$, and the function $b$ is non-increasing in $z$ for fixed $(x, p) \in \Omega \times \mathbf{R}^{N}$. The, if either

(i) the vector function $A$ is independent of $z$; or

(ii) the function $b$ is independent of $p$.

It follows that $u \leq v$ in $\Omega$.

Now, we consider the general equation

$$
\triangle_{\mathfrak{m}} \mathfrak{u}-\psi(x, \mathfrak{u}, \nabla \mathfrak{u})=0 \quad x \in \Omega .
$$


Definition 2.1. Let $1<k \leq+\infty$, functions $\overline{\mathrm{u}}, \underline{u} \in W^{1, k}(\Omega)$ are called the weak upper and lower solutions of (2.1), respectively, if

$$
\psi(\cdot, \overline{\mathrm{u}}(\cdot), \nabla \overline{\mathrm{u}}(\cdot)) \in \mathrm{L}^{\mathrm{k}^{\prime}}(\Omega), \psi(\cdot, \underline{\mathrm{u}}(\cdot), \nabla \underline{\mathrm{u}}(\cdot)) \in \mathrm{L}^{\mathrm{k}^{\prime}}(\Omega)
$$

with

$$
k^{\prime}=\left\{\begin{aligned}
\frac{k}{k-1}, & k<\infty \\
1, & k=\infty
\end{aligned}\right.
$$

and

$$
\begin{aligned}
& \int_{\Omega}|\nabla \bar{u}|^{m-2} \nabla \bar{u} \nabla v \mathrm{~d} x \geq-\int_{\Omega} \psi(x, \bar{u}, \nabla \bar{u}) v \mathrm{~d} x, \quad \forall v \in W_{0}^{1, k}(\Omega), \quad v \geq 0 \text { a.e.in } \Omega \\
& \int_{\Omega}|\nabla \underline{\underline{u}}|^{m-2} \nabla \underline{u} \nabla v \mathrm{~d} x \leq-\int_{\Omega} \psi(x, \underline{u}, \nabla \underline{u}) v \mathrm{~d} x, \quad \forall v \in W_{0}^{1, k}(\Omega), \quad v \geq 0 \text { a.e.in } \Omega .
\end{aligned}
$$

If $\underline{u} \leq \overline{\mathfrak{u}}$, we call that they are the ordered weak upper and lower solutions of (2.1).

Firstly, we consider the existence of weak solution to the problem

$$
\begin{cases}\triangle_{\mathrm{m}} u-\psi(x, u, \nabla u)=0, & x \in \Omega, \\ u=\phi(x), & x \in \partial \Omega,\end{cases}
$$

where $\psi(\cdot, u(\cdot), \nabla u(\cdot)) \in \mathrm{L}^{k^{\prime}}(\Omega), \phi \in W^{1, k}(\Omega)$. Assume that $\bar{u} \in W^{1, k}(\Omega)$ is a weak upper solution $\left(\underline{u} \in W^{1, k}(\Omega)\right.$ is a weak lower solution) of (2.1). Here by $\bar{u} \geq \phi(\underline{u} \leq$ $\phi)$ on $\partial \Omega$, we mean $(\phi-u)^{+}:=\max \{\phi-u, 0\} \in W_{0}^{1, k}(\Omega)\left((u-\phi)^{+} \in W_{0}^{1, k}(\bar{\Omega})\right)$. If $\bar{u} \geq \phi(\underline{u} \leq \phi)$ on $\partial \Omega$, we call that $\bar{u}(\underline{u})$ is a weak upper solution (lower solution) of (2.2). If $\underline{u} \leq \bar{u}$ a.e. in $\Omega$, we call that they are ordered.

Lemma 2.2. $([9$, Theorem 4.9$])$. Let $\bar{u}, \underline{u} \in W^{1, k}(\Omega)$ be the ordered weak upper and lower solutions of (2.2), respectively, and $\underline{u} \leq \bar{u}$ a.e. in $\Omega$. Assume that there exists a positive constant $C_{1}$ and a function $h_{1} \in L^{k^{\prime}}(\Omega)$ with $k^{\prime}=k /(k-1)$, such that

$$
|\psi(x, t, \xi)| \leq h_{1}(x)+C_{1}|\xi|^{k-1} \text {, a.e. } x \in \Omega, \forall \xi \in \mathbf{R}^{N}, t \in[\underline{u}, \bar{u}] .
$$

Then there is a weak solution $u \in W^{1, k}(\Omega)$ of the problem $(2.2)$ such that $\underline{u} \leq \boldsymbol{u} \leq$ $\bar{u}$ a.e. in $\Omega$.

Lemma 2.3. Let $\psi \in \mathrm{C}^{\mu}\left(\Omega \times \mathbf{R} \times \mathbf{R}^{\mathrm{N}}\right)$, and $\overline{\mathrm{u}}, \underline{\mathrm{u}} \in \mathrm{W}^{1, \infty}(\Omega)$ be the ordered weak upper and lower solutions of $(2.2), \phi \in \mathrm{C}^{1+\mu}(\bar{\Omega}), 0<\mu<1$, and $\underline{u} \leq \phi \leq$ $\bar{u}$ a.e. in $\Omega$. Assume that there exists constants $k>1, c_{1}>0$, and a function $h_{1} \in C^{\mu}(\Omega) \cap L^{\infty}(\Omega)$, such that (2.3) holds. Then for some $0<\beta<1$, there is a $\mathrm{C}^{1, \beta}$-solution $\mathfrak{u}$ of $(2.2)$ such that $\underline{u} \leq \boldsymbol{u} \leq \overline{\mathfrak{u}}$ in $\Omega$. 
The proof of the lemma 2.3 is similar to [10], so we omit it here.

Definition 2.2. A domain $\Omega$ is called satisfying the uniform outside spherical condition: if there exists a constant $r>0$ such that there exists a sphere $\bar{B}$ whose radius is $r$ in $\mathbf{R}^{\mathbf{N}}$ for any $z \in \partial \Omega$, such that $\bar{B} \cap \bar{\Omega}=\{z\}$.

Noticing that any $\mathrm{C}^{2}$ bounded domain satisfies the uniform outside spherical condition.

Lemma 2.4. ( Theorem 4.2 in [20]) Assume that $\Omega$ is a bounded domain in $\mathbf{R}^{\mathrm{N}}$ satisfying the uniform outside spherical condition, then there exists a series of $\mathrm{C}^{\infty}$ domains $\left\{\Omega_{n}\right\}_{1}^{\infty}$, such that $\bar{\Omega}_{n} \subset \Omega_{n+1} \subset \Omega, \bigcup_{n=1}^{\infty} \Omega_{n}=\Omega$.

Proof of Theorem 1.1. Since $\Omega$ is a $C^{2}$ bounded domain, from Lemma 2.4 we know that there exists a series of $C^{\infty}$ domains $\left\{\Omega_{n}\right\}_{1}^{\infty}$, such that $\bar{\Omega}_{n} \subset \Omega_{n+1} \subset$ $\Omega, \bigcup_{n=1}^{\infty} \Omega_{n}=\Omega$. Now we consider the problem

$$
\begin{cases}\triangle_{\mathrm{m}} \mathrm{u}=\psi(x, u, \nabla u), & x \in \Omega_{\mathrm{n}}, \\ \mathrm{u}=\overline{\mathrm{u}}, & x \in \partial \Omega_{\mathrm{n}} .\end{cases}
$$

Since $\bar{u} \in W^{1, \infty}\left(\Omega_{n}\right)$ and $\underline{u} \in W^{1, \infty}\left(\Omega_{n}\right)$, by (1.5) we see that there is a constant $C_{2}=C_{2}(n)>0$ such that $|\psi(x, t, \xi)| \leq h_{1}(x)+C_{2}+C_{1}|\xi|^{k-1}$, a.e. $x \in \Omega_{n}, \forall \xi \in$ $\mathbf{R}^{\mathrm{N}}, \mathbf{t} \in[\underline{\mathrm{u}}, \overline{\mathrm{u}}]$. It is obvious that $\left.\overline{\mathrm{u}}\right|_{\Omega}$ and $\left.\underline{\underline{u}}\right|_{\Omega}$ are the ordered upper and lower solutions of (2.4), and $h_{1} \in C\left(\Omega_{n}\right)$. By Lemma 2.3, there exists a solution $u_{n} \in$ $C^{1, \beta}\left(\Omega_{n}\right)$ of $(2.4)$ for some $0<\beta<1$ such that $\underline{u} \leq u_{n} \leq \bar{u}$ in $\Omega_{n}$.

Now, we want to apply elliptic interior estimates together with a diagonal process to conclude: $\left\{u_{n}: n \geq 1\right\}$ has a subsequence $\left\{u_{n_{i}}: n_{i} \uparrow \infty\right\}$ such that $\left\{u_{n_{i}}\right\}$ converges to a function $u$ in $\Omega$ (pointwise) and this convergence is in $C^{1}$ on every compact set in $\Omega$. (Therefore, $\mathfrak{u} \in \mathrm{C}^{1}$ and $\operatorname{div}\left(|\nabla \mathfrak{u}|^{\mathrm{m}-2} \nabla \mathfrak{u}\right)=\psi(x, \mathfrak{u}, \nabla \mathfrak{u})$ with $\underline{u}(x) \leq \mathfrak{u}(x) \leq$ $\bar{u}(\chi)$, and this concludes the proof.)

Step 1. On $\Omega_{2},\left\{u_{n}: n \geq 2\right\}$ is uniformly bounded by $\underline{u}(x)$ and $\bar{u}(x)$. Since both $\underline{\mathfrak{u}}(x)$ and $\overline{\mathfrak{u}}(x)$ are bounded functions on $\Omega_{2}$, there exists $M>0$ such that $\|\bar{u}(x)\|_{L^{\infty}\left(\Omega_{\mathfrak{n}}\right)} \leq M$, for all $\mathrm{n} \geq 2$.

From (2.4), $u_{n}$ satisfies

$$
\int_{\Omega_{2}}\left|\nabla u_{n}\right|^{m} \leq \int_{\Omega_{2}} \psi u_{n}
$$

Therefore,

$$
\int_{\Omega_{2}}\left|\nabla u_{n}\right|^{m} \leq M\left(\text { meas } \Omega_{2}\right)^{1 / q^{\prime}} C_{1}\left\|\nabla u_{n}\right\|_{m},
$$

here $1 / q^{\prime}+1 / m=1$, and $C_{1}$ is the Sobolev embedding constant. So, $\left\|u_{n}\right\|_{1, m} \leq C_{2}$. When $1<\mathrm{m}<\mathrm{N}$, the embedding of $W_{0}^{1, m}\left(\Omega_{2}\right)$ in $\mathrm{L}^{\mathrm{Nm} /(\mathrm{N}-\mathrm{m})}\left(\Omega_{2}\right)$ implies that 
$\mathrm{u}_{\mathrm{k}} \in \mathrm{L}^{\mathrm{Nm} /(\mathrm{N}-\mathrm{m})}\left(\Omega_{2}\right)$. Applying Theorem 7.1 in [21, Page 286-287], we obtain the estimate

$$
\sup \left\{\left|u_{n}\right| ; x \in \Omega_{2}\right\} \leq C_{3} \text {, }
$$

here $C_{3}=C_{3}\left(\|\psi\|_{0}\right)$. If $m \geq N$, we get (2.7) from the Sobolev embedding theorem. Using Theorem 1.1 in ([21], Page 251$)$, we see that $u_{n}$ belongs to $C^{\alpha}\left(\bar{\Omega}_{2}\right)$ for some $0<\alpha<1$, and

$$
\left\|u_{n}\right\|_{C^{\alpha}} \leq C_{4}
$$

here $\mathrm{C}_{4}$ is determined by $\mathrm{C}_{3}$. By Proposition 3.7 in [22, Page 806], we also know that $u_{n}$ belongs to $C^{1, \alpha}\left(\overline{\Omega_{2}}\right)$ and

$$
\left\|u_{n}\right\|_{C^{1, \alpha}} \leq C_{5}
$$

here $\mathrm{C}_{5}$ is determined by $\mathrm{C}_{4}$.

From the arguments above we see that there exists $C>0$ such that

$$
\left\|u_{n}\right\|_{C^{1+\alpha}\left(\Omega_{1}\right)} \leq C, \text { for all } n \geq 2
$$

Since the embedding $C^{1+\alpha}\left(\Omega_{1}\right) \rightarrow C^{1}\left(\Omega_{1}\right)$ is compact, there exists a sequence denoted by $\left\{u_{n_{1 j}}\right\}_{j=1,2 \ldots}$ (where $n_{1 j} \uparrow \infty$ ), which converges in $C^{1}\left(\Omega_{1}\right)$. Let $u_{1}(x)=$ $\lim _{j \rightarrow \infty} u_{n_{1 j}}(x)$, for $x \in \Omega_{1}$, then $u_{1}$ is a solution of $(2.1)$ with $\underline{u}(x) \leq u_{1} \leq \bar{u}(x)$.

Step 2. Repeat Step 1 up to the existence of the sequence $\left\{u_{n_{1 j}}\right\}_{j=1,2 \ldots}$ to get a subsequence $\left\{u_{n_{2 i}}\right\}_{i=1,2 \ldots}$ converging in $C^{1}\left(\Omega_{2}\right)$ to a limit $u_{2}$. Then, likewise, $\boldsymbol{u}_{2}$ is a solution of (2.4) and $\left.u_{2}\right|_{\Omega_{1}}=\mathfrak{u}_{1}$. Repeat Step 1 again on $\Omega_{3}, \ldots$, etc. In this way, we obtain a sequence $\left\{u_{n_{n j}}\right\}_{j=1,2 \ldots}$ which converges in $C^{1}\left(\Omega_{n}\right)$ and is a subsequence of $\left\{u_{n_{n-1) j}}\right\}_{j=1,2 \ldots}$. Let $u_{n}=\lim _{j \rightarrow \infty} u_{n_{n j}}$, then, $u_{n}$ is a solution of $(2.4)$ in $\Omega_{n}$ and $\left.u_{n}\right|_{\Omega_{n-1}}=u_{n-1}$.

Step 3. By a diagonal process, $\left\{u_{n_{l l}}\right\}_{l=1,2 \ldots}$ is a subsequence of $\left\{u_{n_{l j}}\right\}_{j=1,2 \ldots}$ for every $l$. Thus, on $\Omega_{n}$ for each $n$ we have

$$
\lim _{l \rightarrow \infty} u_{n_{l l}}=u_{n}
$$

So, if we define $u(x)=\lim _{n \rightarrow \infty} u_{n}(x)$, then $u(x)$ satisfies

$$
\operatorname{div}\left(|\nabla u|^{m-2} \nabla u\right)=\psi(x, u, \nabla u)
$$

and $\underline{u} \leq \mathfrak{u}(x) \leq \bar{u}$ (since $\underline{u} \leq u_{n}(x) \leq \bar{u}$ ) for every $n$. This completes the proof of Theorem 1.1. 


\section{Proofs of Theorems 1.2 and $\mathbf{1 . 3}$}

To get the existence of large solutions, we first find the blow-up lower and super solutions. Furthermore, if the blow-up lower solution and upper solution have the same blow-up rate near the boundary, we could get the asymptotic behavior of large solutions near the boundary. The idea of this section mainly comes from [10],[12][14].

\section{1 blow-up rate}

In order to get the asymptotic behavior of large solutions in the general domain, we first study the radically symmetric case:

$$
\left\{\begin{array}{l}
\left(\Phi_{m}\left(v^{\prime}\right)\right)^{\prime}+\frac{N-1}{r} \Phi_{p}\left(v^{\prime}\right)=a(r)(R-r)^{\gamma} v^{p}\left(1+\left|v^{\prime}\right|^{q}\right), r \in(0, R), \\
v^{\prime}(0)=0, \quad \lim _{r \rightarrow R} v(r)=\infty,
\end{array}\right.
$$

where $\Phi_{m}(u)=|u|^{m-2} u, m \geq 2$. To ascertain the blow-up rate of the solution of (3.1) at $\mathrm{R}>0$, we first find out the blow-up rate of the following one-dimensional problem

$$
\left\{\begin{array}{l}
\left(\Phi_{m}\left(u^{\prime}\right)\right)^{\prime}=a(r)(R-r)^{\gamma} u^{p}\left(1+\left|u^{\prime}\right|^{q}\right), r \in(0, R) \\
u^{\prime}(0)=0, \quad \lim _{r \rightarrow R} u(r)=\infty
\end{array}\right.
$$

Set $u(r)=(R-r)^{-\alpha} \psi(r), r \in[0, R]$ for some positive constant $\alpha$ which will be determined later, $\psi(r) \in C^{2}(0, R)$, then the problem (3.2) becomes

$$
\begin{aligned}
\left(\left|u^{\prime}\right|^{m-2} u^{\prime}\right)^{\prime}= & (m-1)\left(\alpha(R-r)^{-\alpha-1} \psi(r)+(R-r)^{-\alpha} \psi^{\prime}(r)\right)^{m-2} \\
& \operatorname{sgn}\left(\alpha(R-r)^{-\alpha-1} \psi(r)+(R-r)^{-\alpha} \psi^{\prime}(r)\right) \\
& {\left[\alpha(\alpha+1)(R-r)^{-\alpha-2} \psi(r)+2 \alpha(R-r)^{-\alpha-1} \psi^{\prime}(r)+(R-r)^{-\alpha} \psi^{\prime \prime}(r)\right] } \\
= & (m-1)(R-r)^{-\alpha-2-(\alpha+1)(m-2)}\left(\alpha \psi(r)+(R-r) \psi^{\prime}(r)\right)^{m-2} \\
& \operatorname{sgn}\left(\alpha(R-r)^{-\alpha-1} \psi(r)+(R-r)^{-\alpha} \psi^{\prime}(r)\right) \\
& {\left[\alpha(\alpha+1) \psi(r)+2 \alpha(R-r) \psi^{\prime}(r)+(R-r)^{2} \psi^{\prime \prime}(r)\right] } \\
= & a(r)(R-r)^{\gamma-\alpha p} \psi^{p}(r)+a(r)(R-r)^{\gamma-\alpha p-(\alpha+1) q} \\
& \psi^{p}(r)\left|\alpha \psi(r)+(R-r) \psi^{\prime}(r)\right|^{q}
\end{aligned}
$$

with the boundary condition $\psi(0)=0$, and $\psi(R) \in(0, \infty)$. Therefore, the constant $\alpha$ provides us with the exact blow-up rate of $u$ at $R$. Multiplying (3.3) by $(R-$ $r)^{\alpha+2+(\alpha+1)(m-2)}$ we have 


$$
\begin{aligned}
& (m-1)\left(\alpha \psi(r)+(R-r) \psi^{\prime}(r)\right)^{m-2} \operatorname{sgn}\left(\alpha(R-r)^{-\alpha-1} \psi(r)\right. \\
& \left.+(R-r)^{-\alpha} \psi^{\prime}(r)\right)\left[\alpha(\alpha+1) \psi(r)+2 \alpha(R-r) \psi^{\prime}(r)+(R-r)^{2} \psi^{\prime \prime}(r)\right] \\
= & a(r)(R-r)^{\gamma-\alpha p+\alpha+2+(\alpha+1)(m-2)} \psi^{p}(r) \\
& +a(r)(R-r)^{\gamma-\alpha p-(\alpha+1) q+\alpha+2+(\alpha+1)(m-2)} \psi^{p}(r)\left|\alpha \psi(r)+(R-r) \psi^{\prime}(r)\right|^{q} .
\end{aligned}
$$

Assuming $\lim _{r \rightarrow R}(R-r)^{2} \psi^{\prime \prime}(r)=\lim _{r \rightarrow R}(R-r) \psi^{\prime}(r)=0$, we obtain

$$
\alpha=\frac{\gamma+m-q}{p+q+1-m}, \quad \psi(R)=\left(\frac{\alpha^{m-1-q}(\alpha+1)(m-1)}{a(R)}\right)^{\frac{1}{p+q+1-m}} .
$$

Theorem 3.1. Assume that $R>0, a \in C([0, R] ;(0, \infty)), m \geq 2, \gamma \geq 0, p, q>$ 0 , and $\gamma+m-q>0, p+q>m-1$. Let $\alpha$ and $\psi(R)$ be defined by (3.4). Then for each $\epsilon>0$, the problem (3.1) has at least one nonnegative $C^{1}$-solution $v_{\epsilon}$ satisfying

$$
1-\epsilon \leq \liminf _{r \rightarrow R} \frac{v_{\epsilon}(r)}{\psi(R)(R-r)^{-\alpha}} \leq \lim _{r \rightarrow R} \sup \frac{v_{\epsilon}(r)}{\psi(R)(R-r)^{-\alpha}} \leq 1+\epsilon .
$$

Therefore, for each $x_{0} \in \mathbf{R}^{\mathrm{N}}, \mathrm{m} \geq 2$, the function

$$
u_{\epsilon}(x):=v_{\epsilon}(r) \text { with } r:=\left|x-x_{0}\right|
$$

provides us with a radially symmetric nonnegative solution of the problem

$$
\begin{cases}\triangle_{\mathrm{m}} \mathrm{u}=\mathrm{a}(\mathrm{r}) \mathrm{d}^{\gamma}(x) \mathrm{u}^{\mathrm{p}}\left(1+|\nabla u|^{q}\right), & x \in \mathrm{B}_{\mathrm{R}}\left(x_{0}\right), \\ \mathrm{u}=\infty, & x \in \partial \mathrm{B}_{\mathrm{R}}\left(x_{0}\right),\end{cases}
$$

satisfying

$$
1-\epsilon \leq \lim _{d(x) \rightarrow 0} \inf \frac{u_{\epsilon}(x)}{\psi(R) d^{-\alpha}(x)} \leq \lim _{d(x) \rightarrow 0} \sup \frac{u_{\epsilon}(x)}{\psi(R) d^{-\alpha}(x)} \leq 1+\epsilon
$$

where $d(x):=\operatorname{dist}\left(x, \partial B_{R}\left(x_{0}\right)\right)=R-\left|x-x_{0}\right|=R-r$.

Proof. Firstly, we show that, for each $\epsilon>0$ sufficiently small, there exists a constant $A_{\epsilon}>0$, for each $A>A_{\epsilon}$,

$$
\bar{v}_{\epsilon}(r):=A+B_{+}\left(\frac{r}{R}\right)^{2}(R-r)^{-\alpha}
$$

provides us with a positive upper solution of (3.1), where $\alpha$ is defined in(3.4),

$$
B_{+}=(1+\epsilon)\left(\frac{\alpha^{m-1-q}(\alpha+1)(m-1)}{a(R)}\right)^{\frac{1}{p+q+1-m}} .
$$


Indeed, $\bar{v}_{\epsilon}^{\prime}(0)=0$ and $\lim _{\mathrm{r} \rightarrow \mathrm{R}} \bar{v}_{\epsilon}(\mathrm{r})=\infty$. Thus, $\bar{v}_{\epsilon}$ is an upper solution of (3.1) if and only if

$$
\begin{aligned}
& \left(\frac{B_{+}}{R^{2}}\right)^{m-1}\left[2(m-1)(R-r)^{2}+4(m-1) \alpha r(R-r)+(m-1) \alpha(\alpha+1) r^{2}\right. \\
+ & \left.2(N-1)(R-r)^{2}+\alpha(N-1) r(R-r)\right]\left|2 r(R-r)^{2}+\alpha r^{2}\right|^{m-2} \\
\leq & a(r)(R-r)^{\gamma-\alpha p+\alpha+2+(\alpha+1)(m-2)}\left(A(R-r)^{\alpha}+B_{+}\left(\frac{r}{R}\right)^{2}\right)^{p}\left((R-r)^{(\alpha+1) p}\right. \\
+ & \left.\frac{2 B_{+}}{R^{2}} r(R-r)+\left.\alpha B_{+}\left(\frac{r}{R}\right)^{2}\right|^{q}\right) .
\end{aligned}
$$

Note that $\gamma-\alpha p+\alpha+2+(\alpha+1)(m-2)=0$, at $r=R,(3.8)$ becomes

$$
(m-1) \alpha^{m-1}(\alpha+1) B_{+}^{m-1} \leq B_{+}^{p+q} \alpha^{q} a(R)
$$

which is valid if and only if

$$
B_{+} \geq\left(\frac{\alpha^{m-1-q}(\alpha+1)(m-1)}{a(R)}\right)^{\frac{1}{p+q+1-m}}
$$

Therefore, according to the choice of $\mathrm{B}_{+}$, inequality (3.8) is satisfied in a left neighborhood of $r=R$, say $(R-\delta, R]$ for some $\delta=\delta(\epsilon)>0$. Finally, by choosing $A$ sufficiently large, it is clear that the inequality is satisfied in the whole interval $[0, R]$ since $p>0$ and $a$ is away from zero. This concludes the proof of the claim above.

Now we will construct a suitable lower solution for problem (3.1). We claim that for each sufficiently small $\epsilon>0$, there exists $C<0$ such that

$$
\underline{v}_{\epsilon}(r):=\max \left\{0, C+B_{-}(r / R)^{2}(R-r)^{-\alpha}\right\}
$$

provides us with a nonnegative lower solution of (3.1), here

$$
B_{-}=(1-\epsilon)\left(\frac{\alpha^{m-1-q}(\alpha+1)(m-1)}{a(R)}\right)^{\frac{1}{p+q+1-m}}
$$

Indeed, $\underline{v}_{\epsilon}$ is a lower solution of $(3.1)$ where

$$
C+B_{-}\left(\frac{r}{R}\right)^{2}(R-r)^{-\alpha} \geq 0
$$

which implies 


$$
\begin{aligned}
& \left(\frac{B_{-}}{R^{2}}\right)^{m-1}\left[2(m-1)(R-r)^{2}+4(m-1) \alpha r(R-r)+(m-1) \alpha(\alpha+1) r^{2}\right. \\
& \left.+2(N-1)(R-r)^{2}+\alpha(N-1) r(R-r)\right]\left|2 r(R-r)^{2}+\alpha r^{2}\right|^{m-2} \\
\geq & a(r)(R-r)^{\gamma-\alpha p+\alpha+2+(\alpha+1)(m-2)}\left(C(R-r)^{\alpha}+B_{-}\left(\frac{r}{R}\right)^{2}\right)^{p}(R-r)^{(\alpha+1) p} \\
& \left.+\left|\frac{2 B_{-}}{R^{2}} r(R-r)+\alpha B_{-}\left(\frac{r}{R}\right)^{2}\right|^{q}\right) .
\end{aligned}
$$

Now, for each $C<0$, we can find the constant $z=Z(C) \in(0, R)$, such that

$$
C+B_{-}\left(\frac{r}{R}\right)^{2}(R-r)^{-\alpha}<0 \text { if } r \in[0, Z(C)),
$$

and

$$
C+B_{-}\left(\frac{r}{R}\right)^{2}(R-r)^{-\alpha}>0 \text { if } r \in(Z(C), R) .
$$

Moreover, $\mathrm{Z}(\mathrm{C})$ is decreasing and

$$
\lim _{C \rightarrow-\infty} Z(C)=R, \lim _{C \rightarrow 0} Z(C)=0 .
$$

At $r=R,(3.12)$ becomes into

$$
(m-1) \alpha^{m-1}(\alpha+1) B_{-}^{m-1} \geq B_{-}^{p+q} \alpha^{q} a(R),
$$

which is valid if and only if

$$
B_{-} \leq\left(\frac{\alpha^{m-1-q}(\alpha+1)(m-1)}{a(R)}\right)^{\frac{1}{p+q+1-m}}
$$

Therefore, by making the choice (3.10), inequality (3.11) is satisfied in a left neighborhood of $r=R$, say $(R-\delta, R]$ for some $\delta=\delta(\epsilon)>0$. Moreover, thanks to (3.12), there exists $C<0$, such that $Z(C)=R-\delta(\epsilon)$. For this choice of $C, \underline{v}_{\epsilon}$ provides us with a weak lower solution of (3.1).

Since $\bar{v}_{\epsilon}(r), \underline{v}(r) \in W^{1, \infty}(0, R)$ are the ordered weak lower and upper solutions of (3.1) and are bounded on any closed subdomain of $[0, R)$, it is easy to see (1.5) holds owing to $p, q>0$. So the existence of a $C^{1}$-solution $u$ of (3.1) is followed by Theorem 1.1, and

$$
\underline{v}_{\epsilon}(r) \leq u \leq \bar{v}_{\epsilon}(r) \text { in } \Omega
$$

Finally, since

$$
\lim _{r \rightarrow R} \frac{\bar{v}_{\epsilon}(r)}{B_{+}(R-r)^{-\alpha}}=\lim _{r \rightarrow R} \frac{\underline{v}_{\epsilon}(r)}{B_{-}(R-r)^{-\alpha}}=1
$$

where $\mathrm{B}_{+}$and $\mathrm{B}_{-}$are the constants defined through (3.7)and (3.10), one can easily deduce the remaining assertions of Theorem 3.1. The proof is completed. 


\subsection{Proofs of Theorems $\mathbf{1 . 2}$ and $\mathbf{1 . 3}$}

Proof of Theorem 1.2. For $n \geq 1$, we consider the following problem

$$
\begin{cases}\triangle_{\mathrm{m}} \mathrm{u}=\mathrm{b}(\mathrm{x}) \mathrm{u}^{\mathrm{p}}\left(1+|\nabla \mathrm{u}|^{\mathfrak{q}}\right), & x \in \Omega, \\ \mathrm{u}=\mathrm{n}, & x \in \partial \Omega .\end{cases}
$$

It is obvious that the function $\psi(x, t, \xi)=b(x) t^{p}\left(1+|\xi|^{q}\right)$ satisfies the condition (2.3) since $b(x) \in C^{\mu}(\bar{\Omega})$. The constant functions $u(x)=0$ and $u(x)=n$ are the ordered lower and upper solutions of (3.14). By Lemma 2.3, we see that the problem (3.14) has at least one nonnegative solution $u_{n}(x) \in C^{1}(\Omega)$ and it satisfies $0 \leq u_{n}(x) \leq n<n+1$.

By our assumption, $b(x) \geq \beta_{1} d^{\gamma}(x)>0$ in $\Omega$.So, $\underline{u}=u_{n}$ and $\bar{u}=n+1$ are the order lower and upper solutions of the following problem

$$
\begin{cases}\triangle_{\mathrm{m}} \mathrm{u}=\mathrm{b}(x) \mathrm{u}^{\mathrm{p}}\left(1+|\nabla \mathrm{u}|^{\mathfrak{q}}\right), & x \in \Omega, \\ \mathrm{u}=\mathrm{n}+1, & x \in \partial \Omega .\end{cases}
$$

Therefore $u_{n} \leq u_{n+1}$. Now we fix a point $x_{0} \in \Omega$ and consider a small ball $B$ centered at $x_{0}$ and contained properly in $\Omega$. By Theorem 3.1, there exists a nonnegative $C^{1}$ solution $v(x)$ to the problem

$$
\begin{cases}\triangle_{\mathfrak{m}} u=\beta_{1}(\operatorname{dist}(x, \partial B))^{\gamma} u^{p}\left(1+|\nabla u|^{q}\right), & x \in B_{R}\left(x_{0}\right), \\ u=\infty, & x \in \partial B_{R}\left(x_{0}\right)\end{cases}
$$

Since

$$
\begin{aligned}
& \triangle_{\mathfrak{m}} u_{n}-b(x) u_{n}^{p}\left(1+\left|\nabla u_{n}\right|^{q}\right) \\
\leq & \triangle_{\mathfrak{m}} u_{n}-\beta_{1} d^{\gamma}(x) u_{n}^{p}\left(1+\left|\nabla u_{n}\right|^{q}\right) \\
\leq & \triangle_{m} u_{n}-\beta_{1}(\operatorname{dist}(x, \partial B))^{\gamma}(x) u_{n}^{p}\left(1+\left|\nabla u_{n}\right|^{q}\right), \quad x \in B
\end{aligned}
$$

and $u_{n}(x) \leq v(x)=\infty$ on $\partial B$, by the Comparison principle (Lemma 2.1), we have $u_{n}\left(x_{0}\right) \leq v\left(x_{0}\right)$ for all $n$. Since $u_{n}$ increases in $\Omega$ as $n$ increases, $u_{n}(x)$ is uniformly bounded on any compact subset of $\Omega$. Standard elliptic regularity arguments show that $\lim _{n \rightarrow \infty} u_{n}(x)=u^{*}(x)$ exists and $u^{*}(x)$ satisfies the differential equation of (1.1). To prove $u^{*}(x)$ is a nonnegative solution of (1.1), it remains to verify $\left.u^{*}(x)\right|_{\partial \Omega}=\infty$. If this is not true, then there exist a nonnegative constant $M$, a sequence $\left\{x_{j}\right\} \subset \Omega$ and $x_{0} \in \partial \Omega$, such that $x_{j} \rightarrow x_{0}$ and $u_{n}\left(x_{j}\right) \leq M$. For any fixed $\mathrm{k}$, note that $u_{n}\left(x_{j}\right) \rightarrow u^{*}\left(x_{j}\right)$ as $n \rightarrow \infty$, it follows that there exists $N_{j}>0$, such that $u_{n}\left(x_{j}\right) \leq 1+M$ for all $n \geq N_{j}$. Note that $u_{n}$ is increasing in $n$, we have $u_{n}\left(x_{j}\right) \leq 1+M$ for every $n>0$. Now fix a $n>1+M$ and let $j \rightarrow \infty$ in the above 
inequality, it follows $u_{n}\left(x_{0}\right) \leq 1+M<n$ since $x_{j} \rightarrow x_{0}$, which is a contradiction with $u_{n}\left(x_{0}\right)=n$. The theorem is proved.

Proof of Theorem 1.3. In view of $\lim _{d(x) \rightarrow 0} \frac{b(x)}{d(x)^{\gamma}}=\beta>0$ and $b(x)>0$ in $\Omega$, it is easy to see that

$$
b(x) \geq \beta_{1} d^{\gamma}(x), \quad x \in \Omega
$$

for some $\beta_{1}>0$. Thus, the nonnegative $C^{1}$-solution of problem (1.1) exists by Theorem 1.2 .

Now we prove the limit (1.6). Since

$$
\lim _{d(x) \rightarrow 0} \frac{b(x)}{d(x)^{\gamma}}=\beta>0,
$$

for any small $\epsilon>0$, there exists $\delta=\delta(\epsilon)>0$, such that for all $x \in \Omega$ with $\mathrm{d}(\mathrm{x})<2 \delta$,

$$
(\beta-\epsilon) d^{\gamma}(x) \leq b(x) \leq(\beta+\epsilon) d^{\gamma}(x) .
$$

Now we define $\Omega_{\delta}=\{x \in \Omega: d(x)<\delta\}$ with $\partial \Omega_{\delta}=\{x \in \Omega: d(x)=\delta\}$ and

$$
\begin{cases}u^{+}(x)=B_{+}(\epsilon)(d(x)-\sigma)^{-\alpha}, & x \in D_{\sigma}^{+}=\Omega_{2 \delta} / \bar{\Omega}_{\sigma}, \\ u^{-}(x)=B_{-}(\epsilon)(d(x)+\sigma)^{-\alpha}, & x \in D_{\sigma}^{-}=\Omega_{2 \delta-\sigma},\end{cases}
$$

where $0<\sigma<\delta$, and

$$
\begin{aligned}
& B_{+}(\epsilon)=(1+\epsilon)\left(\frac{\alpha^{m-1-q}(\alpha+1)(m-1)}{\beta-\epsilon}\right)^{\frac{1}{p+q+1-m}}, \\
& B_{-}(\epsilon)=(1-\epsilon)\left(\frac{\alpha^{m-1-q}(\alpha+1)(m-1)}{\beta+\epsilon}\right)^{\frac{1}{p+q+1-m}} .
\end{aligned}
$$

It is easy to prove that by diminishing $\delta>0$ if necessary, $d(x)$ is a $C^{2}$-function on the domain $\bar{\Omega}_{2 \delta}$ and

$$
\begin{cases}\triangle_{\mathfrak{m}} \mathrm{u}^{+}-\mathrm{b}(x)\left(\mathrm{u}^{+}\right)^{\mathfrak{p}}\left(1+\left|\nabla \mathrm{u}^{+}\right|^{q}\right) \leq 0, & x \in \mathrm{D}_{\sigma}^{+}, \\ \triangle_{\mathfrak{m}} \mathrm{u}^{-}-\mathrm{b}(\mathrm{x})\left(\mathrm{u}^{-}\right)^{\mathfrak{p}}\left(1+\left|\nabla \mathrm{u}^{-}\right|^{q}\right) \geq 0, & x \in D_{\sigma}^{-} .\end{cases}
$$

Let $\mathfrak{u}$ be any nonnegative solution of (1.1) and

$$
M_{1}(\delta)=\max _{d(x) \geq 2 \delta} u(x), M_{2}(\delta)=B_{-}(2 \delta)^{-\alpha} .
$$

We see that

$$
\begin{cases}\mathfrak{u}(x) \leq \mathfrak{u}^{+}(x)+M_{1}(\delta), & x \in \partial D_{\sigma}^{+} \\ \mathfrak{u}_{-}(x) \leq \mathfrak{u}(x)+M_{2}(\delta), & x \in \partial D_{\sigma}^{-}\end{cases}
$$


On the other hand, by $p>0$, we have

$$
\left\{\begin{array}{l}
\triangle_{\mathrm{m}}\left(\mathrm{u}^{+}+\mathrm{M}_{1}(\delta)\right)-\mathrm{b}(\mathrm{x})\left(\mathrm{u}^{+}+\mathrm{M}_{1}(\delta)\right)^{\mathrm{p}}\left(1+\left|\nabla\left(\mathrm{u}^{+}+\mathrm{M}_{1}(\delta)\right)\right|^{\mathrm{q}}\right) \\
<0, \quad x \in \mathrm{D}_{\sigma}^{+}, \\
\triangle_{\mathrm{m}}\left(\mathrm{u}+\mathrm{M}_{2}(\delta)\right)-\mathrm{b}(\mathrm{x})\left(\mathrm{u}+\mathrm{M}_{2}(\delta)\right)^{\mathrm{p}}\left(1+\left|\nabla\left(\mathrm{u}+\mathrm{M}_{2}(\delta)\right)\right|^{\mathrm{q}}\right) \\
<0, \quad x \in \mathrm{D}_{\sigma}^{-} .
\end{array}\right.
$$

Note $(3.15)-(3.18)$, it follows by Lemma 2.1 that

$$
\begin{cases}u(x) \leq u^{+}(x)+M_{1}(\delta), & x \in D_{\sigma}^{+}, \\ u_{-}(x) \leq u(x)+M_{2}(\delta), & x \in D_{\sigma}^{-} .\end{cases}
$$

For any $x \in \Omega_{2 \delta}$, there is a $\sigma^{*}>0$, such that $x \in \mathrm{D}_{\delta}^{+} \cap \mathrm{D}_{\delta}^{-}$for all $0<\sigma \leq \sigma^{*}$. Letting $\sigma \rightarrow 0,(3.19)$ yields

$$
B_{-}(\epsilon) d^{-\alpha}(x) \leq u+M_{2}(\delta) \leq B_{+}(\epsilon) d^{-\alpha}(x)+M_{1}(\delta)+M_{2}(\delta),
$$

which implies

$$
B_{-}(\epsilon) \leq \lim _{d(x) \rightarrow 0} \inf \frac{u_{\epsilon}(x)}{d^{-\alpha}(x)} \leq \lim _{d(x) \rightarrow 0} \sup \frac{u_{\epsilon}(x)}{d^{-\alpha}(x)} \leq B_{+}(\epsilon)
$$

Taking $\epsilon \rightarrow 0,(3.20)$ yields

$$
\lim _{d(x) \rightarrow 0} \frac{u(x)}{d^{-\alpha}(x)}=\left(\frac{\alpha^{m-1-q}(\alpha+1)(m-1)}{\beta}\right)^{\frac{1}{p+q+1-m}},
$$

which is accordance with (1.6).

The final step is to prove the uniqueness. Let $\mathfrak{u}_{1}$ and $\mathfrak{u}_{2}$ be two nonnegative solutions of(1.1), then by(1.6), we have

$$
\lim _{d(x) \rightarrow 0} \frac{u_{1}(x)}{u_{2}(x)}=1
$$

Indeed, for $\theta>0$ arbitrary, set

$$
\omega_{i}=(1+\theta) u_{i}, \text { for } i=1,2 .
$$

It follows that

$$
\lim _{d(x) \rightarrow 0}\left(u_{1}-\omega_{2}\right)(x)=\lim _{d(x) \rightarrow 0}\left(u_{2}-\omega_{1}\right)(x)=-\infty .
$$

When $p \geq m-1$, since $q>0$, we have that

$$
\triangle_{m} \omega_{i}-b(x) \omega_{i}^{p}\left(1+\left|\nabla \omega_{i}\right|^{q}\right)<(1+\theta)^{m-1}\left[\triangle_{m} u_{i}-b(x) u_{i}^{p}\left(1+\left|\nabla u_{i}\right|^{q}\right)\right], \quad x \in \Omega .
$$


Therefore, by lemma 2.1, we infer that

$$
u_{1} \leq(1+\theta) u_{2}, \quad u_{2} \leq(1+\theta) u_{1}, \quad x \in \Omega
$$

Passing to the limit $\theta \rightarrow 0^{+}$in (3.21), we get $\mathfrak{u}_{1}=\mathfrak{u}_{2}$ in $\Omega$. This completes the proof of Theorem1.3.

Received: September 2012. Revised: March 2014.

\section{References}

[1] C. Bandle, E. Giarrosso, Boundary blow-up for semilinear elliptic equations with nonlinear gradient terms, Adv.Differential Equations 1(1996), 133-150.

[2] M. Ghergu, C. Niculescu and V. Rădulescu, Explosive solutions of elliptic equations with absorption and non-linear gradient term, Proceedings of the Indian Academy of Sciences.Mathematical Sciences 112(3)(2002), 441-451.

[3] Zuodong Yang, Existence of explosive positive solutions of quasilinear elliptic equations, Applied Mathematics and Computation 177(2006), 581-588.

[4] Qishao Lu, Zuodong Yang, E.H.Twizell, Existence of entire explosive positive solutions of quasilinear elliptic equations, Applied Mathematics and Computation 148(2004), 359-372.

[5] Cunlian Liu and Zuodong Yang, Existence of large solutions for a quasilinear elliptic problem via explosive sub-supersolutions, Applied Mathematics and Computation 199(2008), 414-424.

[6] Cunlian Liu and Zuodong Yang, Existence of large solutions for quasilinear elliptic problems with a gradient term, Applied Mathematics and Computation 192(2007),533-545.

[7] Cunlian Liu and Zuodong Yang, Boundary blow-up quasilinear elliptic problems of the Bieberbach type with nonlinear gradient terms, Nonlinear Anal. 69(2008), 4380-4391.

[8] Cunlian Liu and Zuodong Yang, A Boundary blow-up for a class of quasilinear elliptic problems with gradient term. J.Appl. Math. Comput. 33(2010), 23-34.

[9] Y. H. Du, Order structure and topological methods in nonlinear PDEs, in: Maximum Principle and Applications, vol. 1, World Scientific, Press, Singapore, 2005. 
[10] Yujuan Chen and Mingxin Wang, Large solutions for quasilinear elliptic equation with nonlinear gradient term, Nonlinear Analysis: Real World Applications 12(2011), 455-463.

[11] J. V. Goncalves and A. Roncaili, Boundary blow-up solutions for a class of elliptic equations on a bounded domain, Appl. Math. Comput. 182(2006), $13-23$.

[12] F-C. Cirstea, Y. H. Du, General uniqueness results and variation speed for blow-up solutions of elliptic equations, Proc. Lond. Math. Soc. 91(2)(2005), 459-482.

[13] M. Delgado, J. Lopez-Gomez and A. Suarez, Singular boundary value problems of a porous media logistic equation, Hiroshima Math. J. 34(2004), 57-80.

[14] J. Lopez, The boundary blow-up rate of large solutions, J.Differential Equations 195(2003), 25-45.

[15] F. Gladiali, G. Porru, Estimates for explosive solutions to p-Laplace equations, in: Progress in Partial Differential Equations, Pont--Mousson, 1997, vol. 1, in: Pitman Res. Notes Math. Ser., vol. 383, Longman, Harlow, 1998, pp. 117-127.

[16] Y. Du, Z. Guo, Boundary blow-up solutions and their applications in quasilinear elliptic equations, J. Anal. Math. 89 (2003) 277-302.

[17] Ahmed Mohammed,Boundary asymptotic and uniqueness of solutions to the p-Laplacian with infinite boundary values.J. Math. Anal. Appl. 325 (2007) 480-489.

[18] G. Diaz, R. Letelier, Explosive solutions of quasilinear elliptic equation: existence and uniqueness, Nonlinear Anal. 20(1993), 97-125.

[19] Zuodong Yang, Bing Xu and Mingzhu Wu, Existence of positive boundary blow-up solutions for quasilinear elliptic equations via sub and supersolutions, Applied Mathematics and Computation 188(1)(2007), 492-498.

[20] A. C. Lazer, P. J. McKenna, On a problem of Bieberbach and Rademacher, Nonlinear Anal. 21(1993), 327-335.

[21] O. A. Ladyzhenskaya,N. N. Ural'tseva, Linear and Quasilinear Elliptic Equations, Academic Press, New York, 1968.

[22] P. Tolksdorf, On the Dirichlet problem for quasilinear equations in domains with conical boundary point, Comm. Partial Differential Equations, 8(7)(1983), 773-817. 
[23] D. Gilbarg, N. S. Trudinger, Elliptic Partial Differential Equations of Second Order,2nd ed., Springer-Verlag, Press, Berlin, 1983.

[24] T. Leonori, Large solutions for a class of nonlinear elliptic equations with gradient terms, Adv. Nonlin. Stud. 7(2007), 237-269.

[25] S.Alarcon, J.Garcia-melian, A. Quaas, Keller-Osserman type conditions for some elliptic problems with gradient terms, J.Diff.Eqns. 252(2012), 886-914. 